\title{
Identification of IQ motif-containing GTPase-activating protein 1 as a regulator of long-term ketosis
}

\author{
Hanna L. Erickson and Sayeepriyadarshini Anakk \\ Department of Molecular and Integrative Physiology, University of Illinois at Urbana-Champaign, Urbana, Illinois, USA.
}

IQ motif-containing GTPase-activating protein 1 (IQGAP1) is a ubiquitously expressed scaffolding protein that integrates multiple cellular processes, including motility, adhesion, and proliferation, but its role in metabolism is unknown. Here, we show that IQGAP1 is induced upon fasting and regulates $\boldsymbol{\beta}$-oxidation of fatty acids and synthesis of ketone bodies in the liver. IQGAP1-null $\left(\right.$ Iqgap $\left.1^{-/}\right)$mice exhibit reduced hepatic PPAR $\alpha$ transcriptional activity, as evidenced during fasting, after ketogenic diet, and upon pharmacological activation. Conversely, we found that the activity of fed-state sensor mTORC1 is enhanced in Iqgap1/-- livers, but acute inhibition of mTOR in Iqgap $1^{1-}$ mice was unable to rescue the defect in ketone body synthesis. However, reexpressing IQGAP1

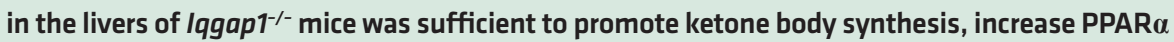
signaling, and suppress mTORC1 activity. Taken together, we uncover what we believe to be a previously unidentified role for IQGAP1 in regulating PPAR $\alpha$ activity and ketogenesis.

Conflict of interest: The authors have declared that no conflict of interest exists.

License: Copyright 2018, American Society for Clinical Investigation.

Submitted: February 2, 2018 Accepted: September 26, 2018 Published: November 2, 2018

\section{Reference information:} JCI Insight. 2018;3(21):e99866. https://doi.org/10.1172/jci. insight.99866.

\section{Introduction}

IQ motif-containing GTPase-activating protein1 (IQGAP1) is a ubiquitously expressed multidomain scaffolding protein that interacts with several binding partners to control diverse cellular processes $(1,2)$, such as cell-cell adhesion via E-cadherin (3), cell motility via Cdc42 and Rac1 (4), and cell proliferation via the Ras/MAP kinase pathway $(5,6)$. More recently, its roles in scaffolding PI3K/AKT signaling as well as insulin receptor and its substrate IRS-1 have been reported $(7,8)$. While all of these cellular processes coordinated by IQGAP1 require energy, the role for IQGAP1 in regulating whole-body energy homeostasis is poorly understood.

To invariably meet cellular energy demands, the mammalian metabolic response has evolved specific programs to store and utilize energy. The liver plays a central role in coordinating these adaptations, such that it promotes glycogen and fatty acid synthesis in the fed state but switches to fat breakdown and ketone body synthesis in the fasted state (9-16). Since IQGAP1 effectively scaffolds to integrate cellular functions, we investigated a role for it in liver metabolism.

We found that IQGAP1 levels were induced upon fasting and that IQGAP1-null (Iqgap $\left.1^{-/-}\right)$mice exhibited reduced fatty acid oxidation and ketogenesis compared with WT mice. Since nuclear receptor PPAR $\alpha$ coordinates these fasting responses $(11,12,17,18)$, we challenged Iqgap $1^{-1}$ mice with ketogenic diet $(\mathrm{KD})$ and found an exaggerated defect in fatty acid oxidation and ketone body synthesis, indicating that PPAR $\alpha$ signaling may be compromised in Iqgap $1^{-/-}$mice. To investigate this further, we pharmacologically activated PPAR $\alpha$ and observed significantly reduced induction in many of its targets when IQGAP1 was deleted.

Further, we examined mTOR, a nutrient sensor, which coordinates lipogenesis $(14,19)$, and found that Iqgap $1^{-/-}$mice have inherently high hepatic mTORC1 activity. Previously, it was shown that chronic mTORC1 could restrict PPAR $\alpha$ signaling $(10,20,21)$, which is relieved during fasting (22). To evaluate this crosstalk, we inhibited mTOR activity with rapamycin and found that short-term mTOR inhibition did not restore the ketogenic defect in Iqgap $1^{-/-}$mice. However, reintroducing IQGAP1 to the livers of Iqgap $1^{-/-}$mice was able to increase expression of PPAR $\alpha$ target genes involved in fatty acid oxidation, reduce $\mathrm{mTORC1}$ activity, and increase ketone body synthesis. Overall, these findings demonstrate what we believe to be a novel role for IQGAP1 in maintaining an appropriate ketogenic response in the liver. 


\section{Results}

IQGAP1 deletion alters the fasting response. IQGAP1 integrates multiple signaling pathways, and its relative abundance largely determines its function as a scaffold (23). To investigate whether IQGAP1 coordinates metabolic signaling, we fasted WT and Iqgap1 ${ }^{-/-}$(24) mice for 24 hours and found a 2-fold induction in IQGAP1 levels in the liver (Figure 1, A and B). The liver is primarily composed of hepatocytes, and we demonstrate that, even though the nonparenchymal cell (NPC) fraction of the liver expressed higher amounts of IQGAP1 transcripts compared with hepatocytes, the overall liver gene expression pattern reflects that of hepatocytes (Supplemental Figure 1A; supplemental material available online with this article; https://doi.org/10.1172/jci.insight.99866DS1). The increase in IQGAP1 expression was specific to the liver and was not observed in white adipose tissue (WAT) (Supplemental Figure 1B). We next tested if the decrease in glucose or growth factor is responsible for IQGAP1 induction and found that glucose or serum starvation of HepG2 cells does not change IQGAP1 expression (Supplemental Figure 1C). Both WT and Iqgap $1^{-/-}$mice lost comparable amounts of body weight and liver weight upon fasting (Table 1). Serum glucose levels also decreased with fasting as expected, but this decrease was exaggerated in Iqgap $1^{-1}$ mice since these mice were hyperglycemic in the fed state and hypoglycemic in the fasted state (Table 1). The observed hyperglycemia in Iqgap $1^{-/-}$mice was consistent with the observed modest insulin resistance under chow (Supplemental Figure 1D) and recently published data that show that loss of IQGAP1 results in poor glucose tolerance (7). Further, we found that the reduction in fasting glucose is not due to lack of gluconeogenic response in the Iqgap $1^{-1-}$ livers (Supplemental Figure 1, E-G).

Normally, serum glucose levels are maintained by switching to an alternate fuel source during prolonged fasting. For instance, the liver switches to utilizing fat, and lipolysis and release of fatty acids from adipose tissue result in elevation of serum fatty acids. WT and Iqgap $1^{-1-}$ mice exhibited a similar percentage increase in serum nonesterified fatty acids (NEFAs), even though the absolute fasting serum NEFA and triglyceride values were significantly lower in Iqgap $1^{-/-}$mice (Table 1). This is because, compared with WT mice, the average serum NEFA and triglyceride levels were lower in Iqgap $1^{-1-}$ mice, even in the fed state. To test if this was secondary to poor fat mobilization, we examined the gonadal white adipose tissue and found that Iqgap $1^{-/-}$mice lost an average $8 \%$ of their visceral adipose by weight upon fasting while the WT mice lost $5 \%$ (Table 1). Histological analysis of gonadal adipose depots did not reveal any differences in adipocyte size (Supplemental Figure 2A). Furthermore, we found comparable expression of lipogenic genes, including Srebp1c, Fasn, Ppary, and starvation response gene Fgf21 as well as lipases, $H s l, L p l$, and Atgl, in adipose tissue of both animals (Supplemental Figure 2, B-D). Additionally, gonadal phospho-HSL levels were induced to a higher extent upon fasting in Iqgap $1^{-/-}$mice compared with WT mice (Supplemental Figure 2F). These data indicate that the adipose tissue expression profile of fat mobilization genes was unaltered in Iqgap ${ }^{-1}$ mice. Additionally, hepatic fat accumulation after fasting increased to a similar extent in both the WT and Iqgap $1^{-/-}$livers (Figure 1C), suggesting that IQGAP1 deletion does not affect fat uptake into hepatocytes.

We then examined if the fatty acids taken up by the liver during fasting are oxidized and converted to ketone bodies, which are essential alternate fuels. Serum levels of $\beta$-hydroxybutyrate, the predominant form of ketone body, were dramatically increased in WT mice upon fasting, but this was blunted in Iqgap $1^{-1-}$ mice (Figure 1D). We also examined serum ketone concentrations after injection with octanoate, a medium-chain fatty acid substrate that can be rapidly converted to ketones (Figure 1E). The increase in serum $\beta$-hydroxybutyrate concentration was lower in Iqgap $1^{-/-}$mice compared with WT mice (Figure $1 \mathrm{~F}$ ). This result demonstrates that, despite substrate availability, Iqgap $1^{-/-}$mice exhibit impaired ketogenesis. Furthermore, induced expression of $\beta$-oxidation genes medium-chain acyl-CoA dehydrogenase $($ Acadm) and enoyl-CoA hydratase/3-hydroxyacyl-CoA dehydrogenase (Ehhadh) as well as FGF21 (Fgf21), a known endocrine signal during ketosis $(25,26)$, was significantly dampened in the absence of IQGAP1 (Figure 1, G-I). These changes corresponded to lower circulating FGF21 levels in Iqgap 1 ${ }^{-/-}$mice (Figure $\left.1 \mathrm{~J}\right)$. These data uncover a role for IQGAP1 in maintaining appropriate hepatic fatty acid metabolism and ketogenesis.

IQGAP1 is crucial to long-term ketogenic adaptation of the liver. Since we localized the metabolic defect in Iqgap $1^{-/-}$mice to decreased hepatic expression of multiple genes involved in the $\beta$-oxidation pathway, we examined long-term ketosis in these animals. To do this, WT and Iqgap $1^{-{ }^{-}}$mice were fed a high-fat, low-carbohydrate $\mathrm{KD}$ for 4 weeks. Both cohorts displayed similar changes in overall body weight, but liver weight was reduced in KD-fed WT but not in Iqgap $1^{-/-}$mice (Supplemental Figure 3, A and B), resulting in a significant difference in the liver-to-body weight ratio between $\mathrm{KD}$-fed WT and Iqgap $1^{-/-}$mice (Figure 2A). Iqgap $1^{-/-}$livers appeared pale (Figure 2B) and microsteatotic (Figure 2C), which corroborated well with the observed increase 
A

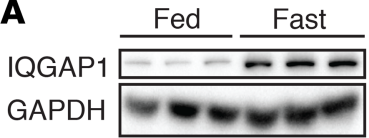

B

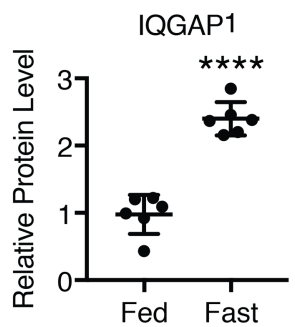

C
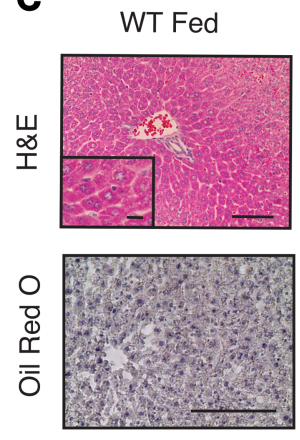

WT Fast
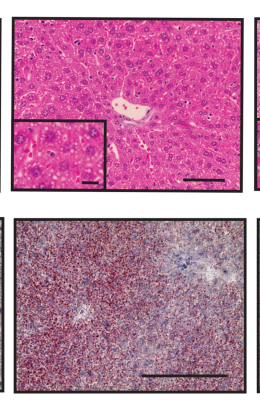

Iqgap $1 \%$ Fed
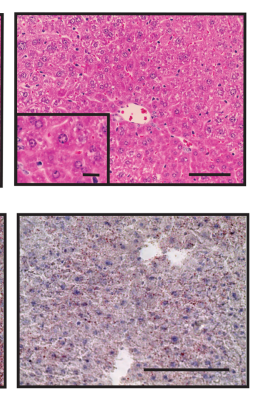

Iqgap $1^{-/}$Fast
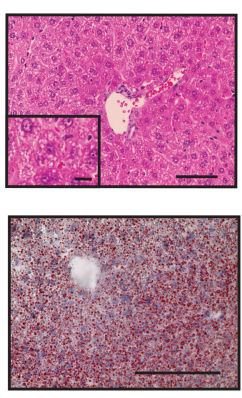

D

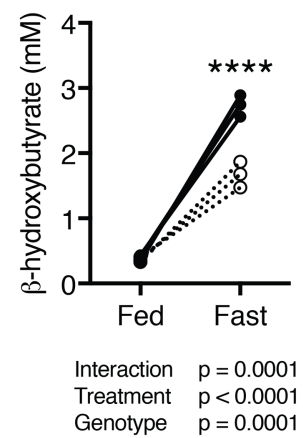

G

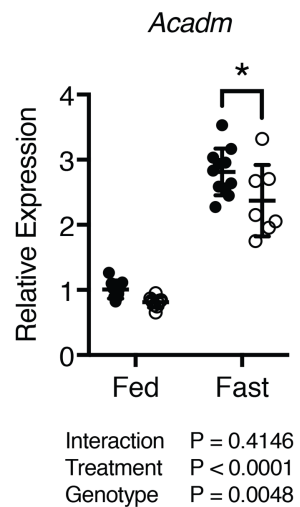

E

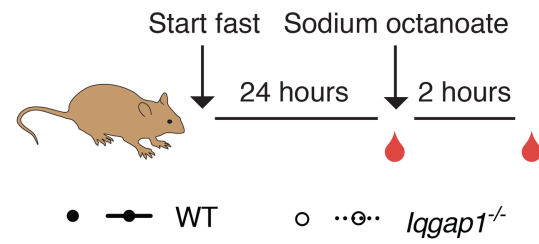

$\mathbf{F}$

Sodium Octanoate

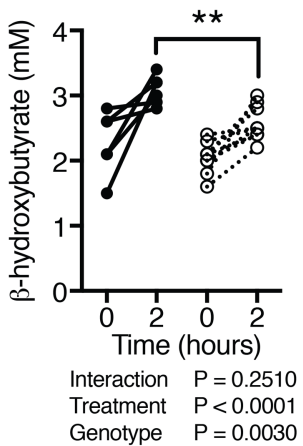

J Serum FGF21

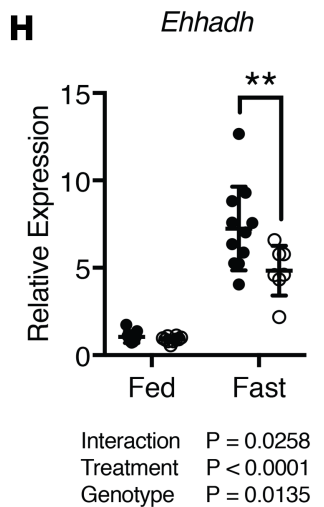

I

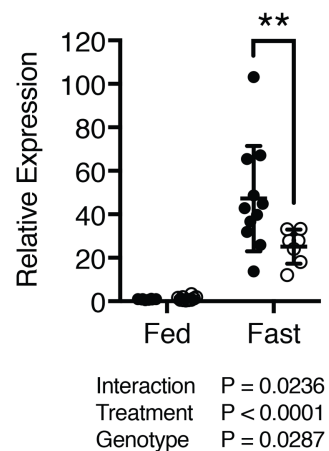

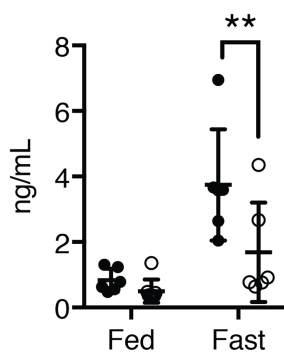

Interaction $\mathrm{P}=0.0631$

Treatment $P=0.0001$

Genotype $\quad P=0.1973$

Figure 1. Hepatic IQGAP1 is induced by fasting and is crucial for the fasting response. Mice were fed ad libitum or fasted for 24 hours. (A) Immunoblots of WT liver extracts indicate increased IQGAP1 expression with fasting. Each lane is a mixture of liver extracts from 2 mice ( $n=6$ mice per group). (B) Quantification of average relative IQCAP1 protein per mouse, measured from 7 Western blots. IQGAP1 levels were normalized to GAPDH using densitometry. Each dot represents a single mouse. (C) Representative images of H\&E staining of liver sections and Oil red 0 staining of frozen liver sections from Iqgap $1^{-/-}$and WT mice ( $n=5-6$ mice per group). Scale bar: $50 \mu \mathrm{m} ; 10 \mu \mathrm{m}$ (inset). (D) Serum $\beta$-hydroxybutyrate levels ( $n=3$ mice per group). (E) Schematic depicting the workflow for measuring ketogenic potential. (F) Serum ketone body levels measured before and after sodium octanoate treatment $(n=6-8$ mice per group). (G-I) Hepatic gene expression of (C) Acadm, (H) Ehhadh, and (I) Fgf21 normalized to Gapdh expression in WT and Iqgap 1/- mice ( $n=6-10$ mice per group). (J) Serum FGF21 levels were measured by ELISA ( $n=6-7$ mice per group). Values are displayed as mean \pm SD. Two-tailed unpaired $t$ test was used to determine significance between 2 groups. Two-way ANOVA with Bonferroni multiple comparisons test was used to determine significance between 2 groups under 2 conditions. ${ }^{*} P<0.05,{ }^{* *} P<0.01,{ }^{* * *} P<0.0001$. See complete unedited blots in Supplemental Figure 7.

in hepatic triglycerides (Figure 2D) compared with that in WT mice. On the other hand, the reduction in serum NEFA and triglycerides was seen in both groups of mice (Figure 2, E and F). Further, the gonadal white adipose tissue size, though originally smaller in Iqgap $1^{-1-}$ mice, increased by a similar proportion in WT and Iqgap $1^{--}$mice (Supplemental Figure 3C) and had similar expression of lipase (Supplemental Figure 3, D-F) and lipogenic (Supplemental Figure 3G) genes, indicating a metabolic defect in the liver. 
Table 1. Metabolic characterization of WT and Iqgap1/- mice

\begin{tabular}{|c|c|c|c|c|}
\hline & \multicolumn{2}{|c|}{ Fed } & \multicolumn{2}{|c|}{ Fast } \\
\hline & WT & Iqgap1/- & WT & Iqgap1/- \\
\hline \multicolumn{5}{|l|}{ Energy balance } \\
\hline Liver weight (g) & $1.03 \pm 0.26$ & $0.95 \pm 0.14$ & $0.80 \pm 0.35$ & $0.66 \pm 0.08^{A}$ \\
\hline Liver-body weight ratio (\%) & $3.75 \pm 0.77$ & $3.82 \pm 0.48$ & $2.72 \pm 0.56^{A}$ & $3.14 \pm 0.22^{A}$ \\
\hline gWAT weight (g) & $0.75 \pm 0.28$ & $0.50 \pm 0.18^{B}$ & $0.67 \pm 0.19$ & $0.38 \pm 0.08^{\mathrm{B}}$ \\
\hline Clucose (mg/dl) & $144.4 \pm 14.2$ & $167.6 \pm 14.5^{B}$ & $96.2 \pm 21.9^{A}$ & $78.6 \pm 18.3^{A, B}$ \\
\hline Triglycerides (mg/dl) & $142.2 \pm 32.3$ & $103.1 \pm 26.4^{B}$ & $103.1 \pm 25.7^{A}$ & $67.1 \pm 4.4^{\mathrm{A}, \mathrm{B}}$ \\
\hline NEFAs (mEq) & $1.61 \pm 0.46$ & $1.19 \pm 0.06$ & $2.28 \pm 0.60^{A}$ & $1.63 \pm 0.18^{B}$ \\
\hline Cholesterol (mg/dl) & $152.2 \pm 33.1$ & $126.4 \pm 26.1$ & $153.1 \pm 41.2$ & $94.4 \pm 15.3^{B}$ \\
\hline \multicolumn{5}{|l|}{ Hepatic parameters } \\
\hline
\end{tabular}

To understand the basis of increased fat accumulation in $\mathrm{KD}$-fed Iqgap $1^{-1-}$ livers, we examined the expression of genes controlling fatty acid breakdown and ketogenesis. We found that induction of carnitine palmitoyltransferase 1A (Cpt1a), $\beta$-oxidation genes Ehhadh and Acadm (but not Hadha), ketogenic genes hydroxymethylglutaryl-CoA synthase 2 (Hmgcs2) and 3-hydroxybutyrate dehydrogenase (Bdh1), Fgf21, and cytochrome P450 4A10 (Cyp4a10), which are all peroxisome proliferator receptor $\alpha$ (Ppara) targets, was all significantly decreased in the absence of IQGAP1 (Figure 3, A-H, and refs. 27-31). Subsequent to the reduced $\beta$-oxidation and ketogenesis, we found lower levels of circulating ketone bodies in $\mathrm{KD}$-fed Iqgap ${ }^{1^{-1}}$ animals compared with those in WT animals (Figure 3I). These results indicate that deletion of IQGAP1 impairs long-term hepatic adaptation to nutritional ketosis (Figure 3J).

Loss of IQGAP1 deregulates PPAR a signaling and mTORC1 activation. The nuclear receptor PPAR $\alpha$ is a critical transcriptional regulator of the fasting and ketogenic response $(25,27)$. In fact, Ppara $^{-/-}$mice are unable to adapt to nutritional challenges and develop fatty liver $(27,32)$. Therefore, we next examined PPAR $\alpha$ activation in the presence and absence of IQGAP1 by treating mice with the PPAR $\alpha$ agonist Wy-14,643 (WY) $(31,33)$. It is interesting to note that Ppara transcript levels were in a higher range upon corn oil (CO) treatment in Iqgap $1^{-/-}$mice and were not induced by WY treatment compared with WT mice (Figure 4A). Several downstream targets of PPAR $\alpha$, including Acadm, Hadha, Hmgcs2, and Bdh1, were not significantly induced in WY-treated Iqgap $1^{-1-}$ mice while Cpt1a and Ehhadh displayed similar induction to that of WY-treated WT mice (Figure 4, B-G). Surprisingly, WY treatment led to higher transcript expres-

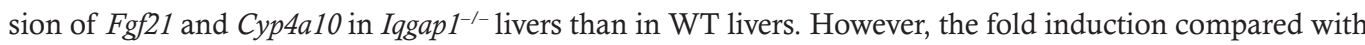
CO-treated mice was similar between the two groups, since the basal levels following CO treatment was higher for these two genes in Iqgap $1^{-/-}$livers (Figure 4, H and I). On the other hand, fed-state serum ketone concentrations did not reveal any increase in response to WY treatment in Iqgap1 $1^{-1-}$ mice (Figure 4J).

Next, we investigated the activity of the fed-state sensor mTORC1 in WT and Iqgap $1^{-1-}$ livers. Activation of mTORC1 was measured by assessing phosphorylation of its two bona fide targets - S6 ribosomal protein and 4E-BP1. S6 phosphorylation at S240/244 and S235/236 sites, along with phosphorylation of 4E-BP1 $(34,35)$, showed a 2-fold increase in Iqgap $1^{-1-}$ livers compared with WT livers upon refeeding (RF) (Figure 5A). Additionally, primary hepatocytes from Iqgap $1^{-/-}$mice exhibited higher S6 phosphorylation (Supplemental Figure 4A), indicative of increased mTORC1 activity when IQGAP1 is absent. Conversely, increasing IQGAP1 levels in the HepG2 cell line was able to decrease S6 phosphorylation (Supplemental Figure 4B). To determine if IQGAP1 could regulate mTORC1 through a direct interaction, we expressed FLAG-tagged IQGAP1 in HEK293T cells and pulled down IQGAP1-interacting proteins using an anti-FLAG antibody. We found higher levels of mTOR pulled down in cells expressing FLAG-IQGAP1 (Supplemental Figure 4C), implying interaction of IQGAP1 with mTOR. As expected, we found that P-mTOR (S2448) levels decreased in the fasted state and were restored upon RF in WT 
A Liver-Body Weight

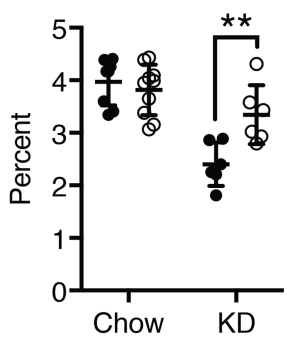

Interaction: $\mathrm{P}=0.0049$ Treatment: $P<0.0001$ Genotype: $\quad P=0.0357$

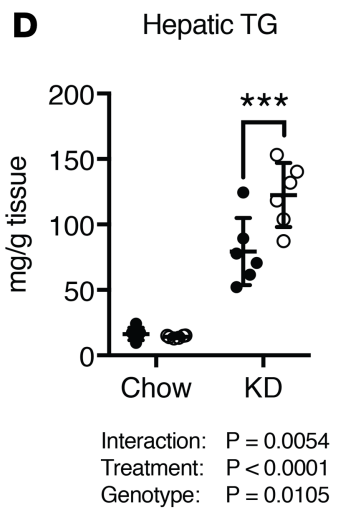

B

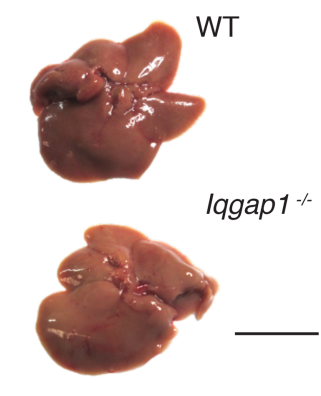

E

Serum NEFA

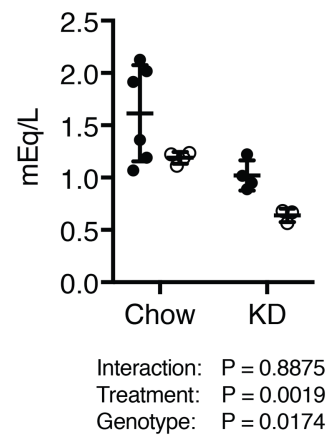

C
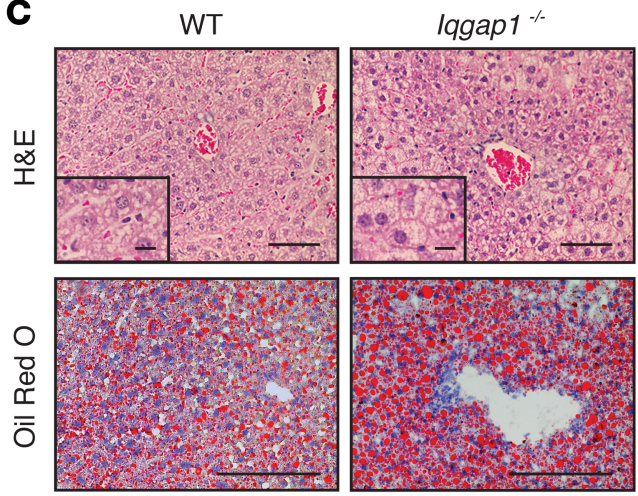

$\mathbf{F}$

Serum TG

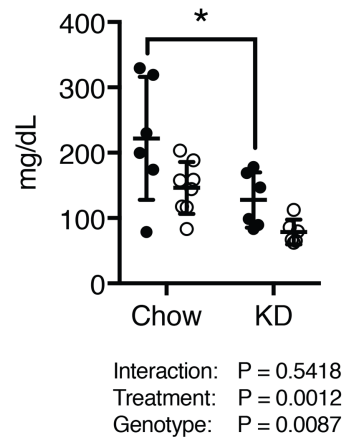

Figure 2. Iqgap $1^{-1-}$ mice accumulate excess hepatic fat when challenged with ketogenic diet. WT and lqgap1 ${ }^{-1-}$ mice were fed ketogenic diet (KD) for 4 weeks and fasted overnight ( $n=6$ mice per group). Control mice were fed normal chow ad libitum ( $n=9-10$ mice per group). (A) Liver weight normalized to total body weight. (B) Gross appearance of livers from KD-fed WT and Iggap1 $1^{-1-}$ mice. Scale bar: $1 \mathrm{~cm}$. (C) Representative images of H\&E-stained and Oil red 0 -stained liver sections from KD-fed mice ( $n=6$ mice per group). Scale bar: $50 \mu \mathrm{m} ; 10 \mu \mathrm{m}$ (inset). (D) Hepatic triglyceride was measured in lqgap $1^{-1-}$ and WT mice ( $n=6$ mice per group). (E and F) Serum NEFA and triglyceride levels ( $n=3-8$ mice per group). Values are displayed as mean \pm SD. Two-way ANOVA with Bonferroni multiple comparisons test was used to determine significance between 2 groups under 2 conditions. ${ }^{*} P<0.05,{ }^{* *} P<0.01,{ }^{* * *} P<0.001$.

mice (Figure 5B). The induction of P-mTOR relative to mTOR was higher in Iqgap $1^{-/-}$mice, validating increased mTORC1 activity in the absence of IQGAP1. To identify the mechanism responsible for higher activity of mTORC1, we examined upstream signals, such as AKT, which can promote mTORC1 activity, and AMPK, which can inhibit activity. Compared with WT livers, Iqgap $1^{-/-}$livers in the RF state exhibited higher P-AKT (S473) levels, whereas the changes in P-AMPK relative to AMPK were similar (Figure 5B). The increase in AKT signaling is intriguing, since it has been shown that Iqgap $1^{-1-}$ livers exhibit a blunted increase in P-AKT levels 10-15 minutes after insulin treatment (7, 8). Since RF experiments were performed 120 minutes after feeding and not immediately, WT mice did not show a robust increase in P-AKT. It is possible that this in turn may exaggerate the P-AKT levels observed in Iggap $1^{-1}$ livers. Nonetheless, the increase in P-AKT levels is consistent with higher mTORC1 activity.

To confirm this increase in mTORC1 activation, we analyzed Srebp1c and fatty acid synthase (Fasn) expression levels, which are suppressed in livers with chronic mTORC1 activation (14), and found them significantly downregulated in Iqgap $1^{-1-}$ livers (Figure 5, C and D) (36). RF did not alter Srebp1c transcript levels but induced Fasn expression in the WT mice. mTOR inhibition in RF mice dramatically reduced expression of Srebp1c gene in both WT and Iqgap $1^{-/-}$mice, indicating that mTOR activation is important to maintain this regulator of lipogenesis (Figure 5C). On the other hand, Fasn transcript levels were reduced with rapamycin treatment in WT mice but not in Iqgap $1^{1^{--}}$mice (Figure 5D). Notably, in Iqgap $1^{-1-}$ mice the levels of Fasn gene expression increased upon mTOR inhibition, and we suggest that this is subsequent to the transient relieving of chronic mTOR activation in these animals. Taken together, these data indicate that IQGAP1 maybe important to maintain proper hepatic mTORC1 activity and function. 
A

Cpt1a

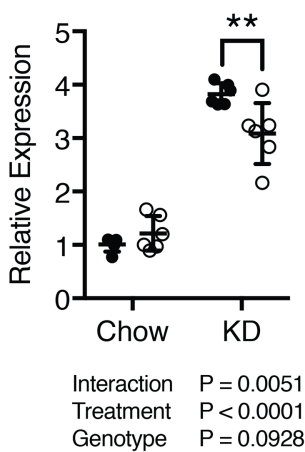

E

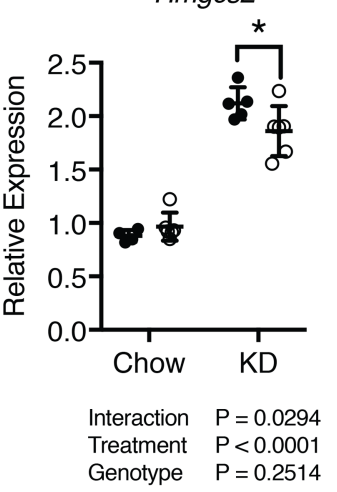

I Serum Ketones

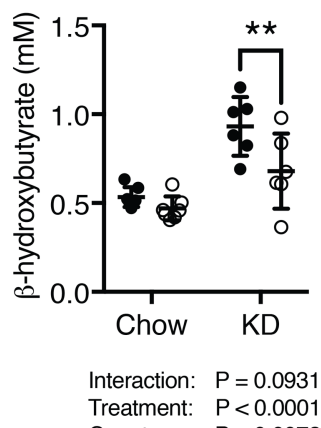

B

Acadm

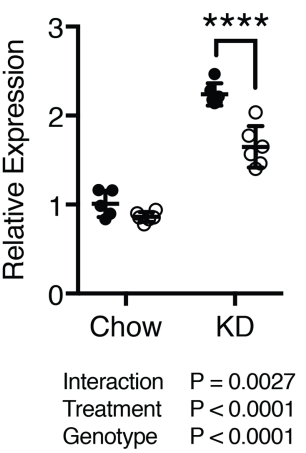

$\mathbf{F}$

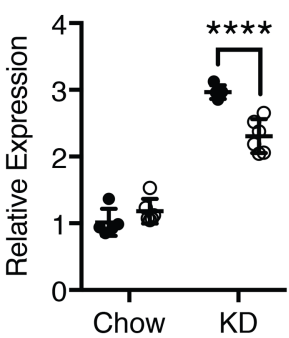

Interaction $\mathrm{P}=0.0001$

Treatment $P<0.0001$

Genotype $\quad P=0.0087$
C Ehhadh

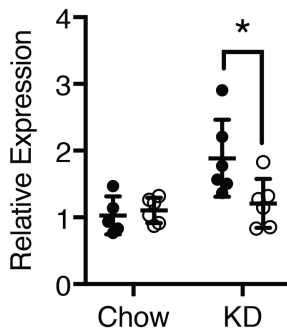

Interaction $\mathrm{P}=0.0312$

Treatment $P=0.0078$

Genotype $\quad P=0.0785$

G

Fgf21

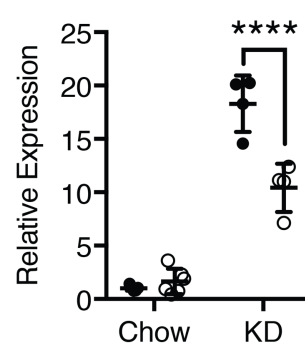

Interaction $\mathrm{P}<0.0001$

Treatment $\quad P<0.0001$

Genotype $\quad P=0.0004$
D Hadha

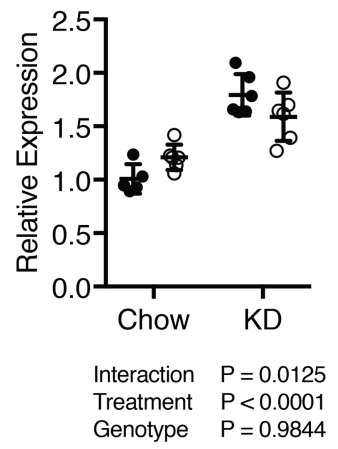

H Cyp4a10

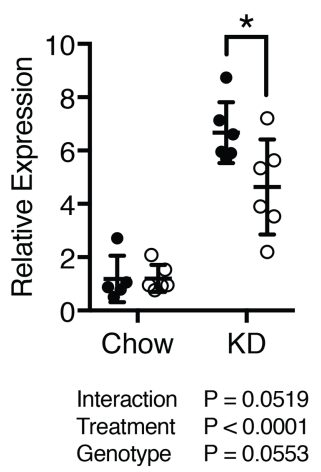

- WT

- lqgap $^{-/}$

Figure 3. Ketogenesis is significantly reduced when IQGAP1 is deleted. (A-H) Hepatic gene expression of $\beta$-oxidation genes (A) Cpt1a, (B) Acadm, (C) Ehhadh, and (D) Hadha and ketogenesis genes (E) Hmgcs2, (F) Bdh1, and (C) Fgf21 and (H) $\omega$-oxidation gene Cyp4a10. All gene expression was normalized to Gapdh expression. (I) Serum $\beta$-hydroxybutyrate levels ( $n=6-7$ mice per group). (J) Schematic depicting genes with altered expression in lqgap ${ }^{1 /-}$ mice in blue and their position within the fatty oxidation and ketogenesis pathways. Values are displayed as mean \pm SD. Two-way ANOVA with Bonferroni multiple comparisons test was used to determine significance between 2 groups under 2 conditions. ${ }^{*} P<0.05,{ }^{* *} P<0.01,{ }^{* * * *} P<0.0001$.

Short-term mTOR inhibition does not alleviate the ketogenic defect in Iqgap1 $1^{-1-}$ mice. We next tested if inhibiting mTOR with rapamycin can reverse any of the $\beta$-oxidation defects observed in Iqgap $1^{-/-}$mice. We first ensured that loss of IQGAP1 did not affect mTORC1 inhibition by rapamycin in vivo (Supplemental Figure 5A). Hepatic triglyceride concentration was reduced when mTOR was inhibited in the RF state (Supplemental Figure 5B), while serum triglycerides remained unaltered in WT mice (Supplemental Figure 5C). No alteration in hepatic triglyceride levels was observed in IQGAP1-deleted livers. However, serum triglyceride concentrations were lower in the fasted state and were modestly elevated in the RF state but significantly lower in the rapamycin-treated RF condition (Supplemental Figure 5C). Serum NEFA levels on the other hand were high during fasting, which indicates fat mobilization from adipose tissue, and this was reduced upon RF. This decrease in NEFA levels 
A

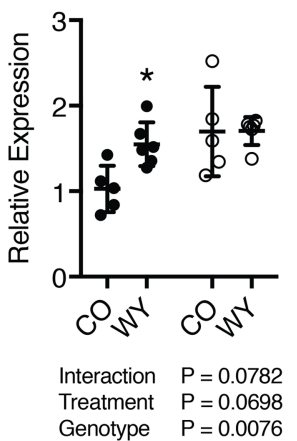

E

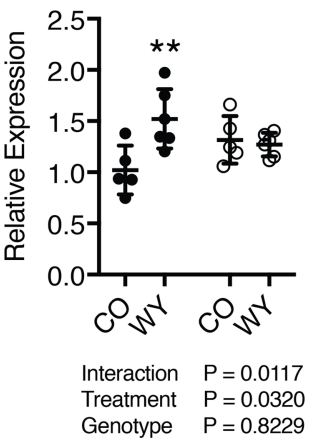

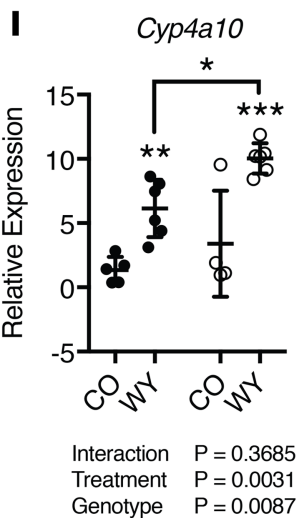

C

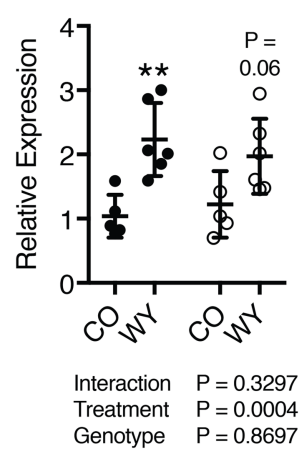

G

Ehhadh

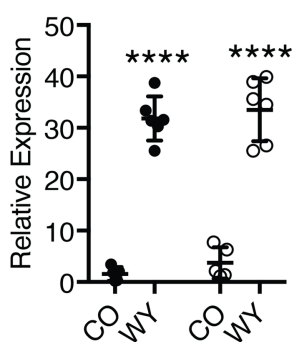

Interaction $\mathrm{P}=0.9003$

Treatment $P<0.0001$

Genotype $\quad P=0.3067$
D Hmgcs2

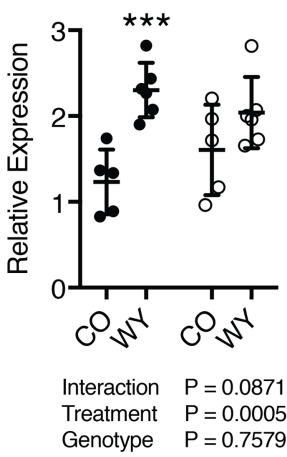

H

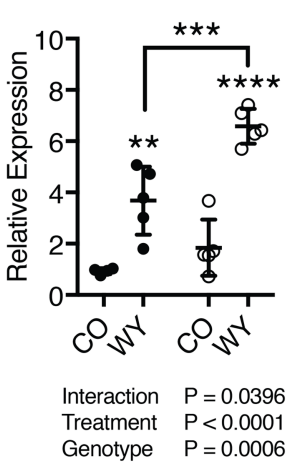

- WT

○ Iqgap 1 $^{-1}$

Figure 4. PPAR $\alpha$ activation is dysregulated in lqgap1 ${ }^{-/-}$livers. WT and lqgap1 $1^{-/-}$mice were administered vehicle (CO) or Wy-14,643 (WY) daily for 4 days. (A-E) Hepatic gene expression of (A) Ppara, $\beta$-oxidation genes (B) Acadm and (C) Hadha, and ketogenesis genes (D) Hmgcs2 and (E) Bdh1 shows decreased induction in lqgap1-/- animals. (F and $\mathbf{G})$ Hepatic gene expression of $\beta$-oxidation genes (F) Cpt1a and (C) Ehhadh shows similar induction. ( $\mathbf{H}$ and $\mathbf{I})$ Hepatic gene expression of $(\mathbf{H})$ Fgf21 and (I) $\omega$-oxidation gene Cyp4a10. Expression was normalized to Gapdh expression ( $n=5-6$ mice per group). (J) Serum $\beta$-hydroxybutyrate levels in CO- and WY-treated mice ( $n=5-6$ mice per group). Values are displayed as mean \pm SD. Two-way ANOVA with Bonferroni multiple comparisons test was used to determine significance between 2 groups under 2 conditions. ${ }^{*} P<0.05,{ }^{* *} P<0.01,{ }^{* *} P<0.001$, and ${ }^{* * *} P<0.0001$

upon $\mathrm{RF}$ was not dependent on mTORC1, as rapamycin treatment did not affect the reduction in either WT or Iqgap $1^{-/-}$animals (Supplemental Figure 5D). RF, as expected, reduced Ppara expression, and rapamycin levels were not altered in both WT and Iqgap $1^{-/}$livers (Supplemental Figure 5E). Expression of PPAR $\alpha$ target genes involved in $\beta$-oxidation, such as Cpt1a, Ehhadh, and Acadm (Supplemental Figure 5, F-H), was reduced upon RF in WT mice. Iggap $1^{---}$livers displayed lower levels of PPAR $\alpha$ target genes both under fasted and RF conditions, and rapamycin treatment did not alter their expression pattern. These results suggest that mTOR-independent mechanisms may contribute toward $\beta$-oxidation in the liver and ketogenesis in Iqgap $1^{-/-}$livers. 
A

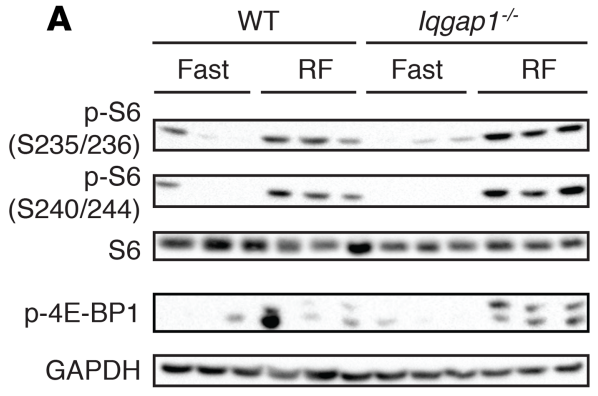

$$
\text { B }
$$

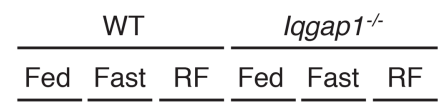

P-mTOR

(S2448)

mTOR

P-AKT

(S473)

Pan AKT

P-AMPKa

(T172)

AMPKa

GAPDH

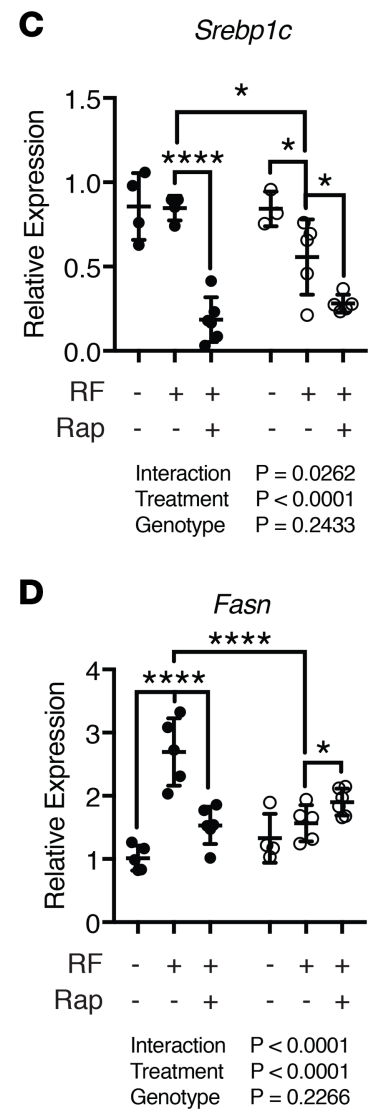

Figure 5. Effect of mTOR inhibition in Iqgap $1^{-1-}$ mice. WT and Iqgap1 $1^{-1-}$ mice were fasted for 24 hours or fasted 24 hours and then given chow for 2 hours (RF). (A) Immunoblot of WT and Iqgap1/-- liver extracts from fasted and RF mice. Each lane is a mixture of liver extracts from 2 mice. (B) Immunoblot of WT and Iqgap $1^{-/-}$liver extracts from ad libitum fed, fasted, and RF mice. Each lane is a mixture of liver extracts from 2 mice. (C and D) Hepatic gene expression of (C) Srebp1c and (D) Fasn in fasted and RF WT and Iqgap1/- mice and mice treated with $10 \mathrm{mg} / \mathrm{kg}$ rapamycin 1 hour prior to refeeding (Rap+RF) shows an interaction between treatment and genotype effects. Gene expression normalized to Gapdh expression ( $n=3-6$ mice per group). Values are displayed as mean \pm SD. Two-way ANOVA with Bonferroni multiple comparisons test was used to determine significance between 2 groups under 3 conditions. ${ }^{*} P<0.05,{ }^{* * * *} P<0.0001$. See complete unedited blots in Supplemental Figure 7.

Restoration of hepatic IQGAP1 expression reverses PPAR $\alpha$ and $m$ TORC1 effects. Finally, we examined if reexpressing IQGAP1 specifically in the livers of Iqgap1 $1^{-/-}$mice (Supplemental Figure 6A) is sufficient to restore metabolic gene expression. We confirmed that the adenoviral IQGAP1 injections did not result in IQGAP1 expression in the gonadal adipose depots (Supplemental Figure 6B). Compared with adenoviral GFP control, adenoviral IQGAP1 resulted in reduced hepatic mTORC1 activity (Figure 6A) and increased serum ketone concentrations in fasted state (Figure 6B) in Iqgap $1^{-/-}$mice. Importantly, reintroducing IQGAP1 was sufficient to induce PPAR $\alpha$ target genes (Figure 6, C and D), indicating that IQGAP1 is necessary to maintain fatty acid oxidation and ketogenesis. Taken together, these data reveal that IQGAP1 levels are crucial to maintaining PPAR $\alpha$ and mTORC1 activity and thus modulate ketogenesis (Figure 6E).

\section{Discussion}

The liver plays a central role in nutrient metabolism, and several transcriptional, posttranslational, and allosteric regulators that orchestrate this process have been identified (11, 15-19, 37). Scaffolding proteins can integrate different signals, but their metabolic roles are yet to be fully elucidated. In this study, we examined a role for IQGAP1, a multidomain-containing scaffolding protein that interacts with numerous signaling molecules, including calmodulin (38), MAPK (6), PI3K (11), AKT (3), and forkhead box protein $\mathrm{O} 1$ (39), in coordinating the hepatic response to long-term ketosis. While its role in cellular signaling has been well studied $(1,2)$, the role for IQGAP1 in hepatic metabolism is poorly understood.

We found increased IQGAP1 expression in the liver upon fasting that was not secondary to the decrease in serum glucose levels (Supplemental Figure 1C). Instead, we identified that the IQGAP1 increase was associated with fatty acid oxidation and ketogenesis, since Iqgap $1^{-1-}$ mice showed defects in these pathways and reexpressing hepatic IQGAP1 could reverse the PPAR $\alpha$ signaling defect. Despite being slightly more hypoglycemic than the WT mice during fasting, Iqgap $1^{-/-}$mice did not show alterations in the gluconeogenic response (Supplemental Figure 1, D-G), which could be attributed to intact expression of Pgcla, a key regulator of gluconeogenesis (40), in the livers of Iqgap $1^{-1-}$ mice. Rather, this hypoglycemia may be secondary to lower serum ketone body levels. In particular, we found that IQGAP1 was required to maintain long-term ketogenesis, since Iqgap $1^{-1-}$ mice displayed a poor ability to break down fat and synthesize ketone bodies when we challenged 
A

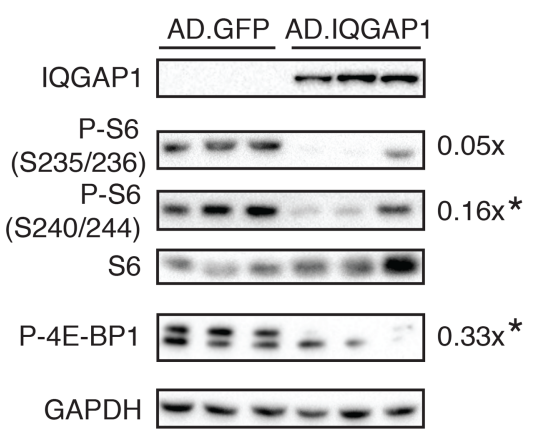

D

Ehhadh

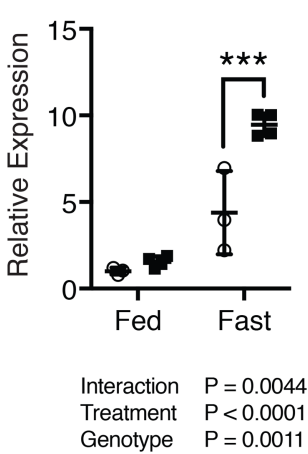

Cyp4a10

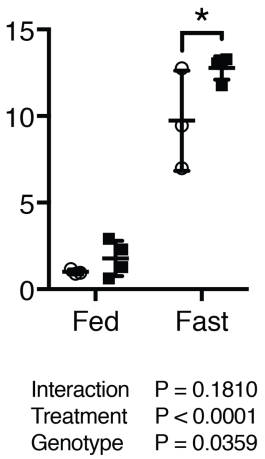

B Serum Ketones

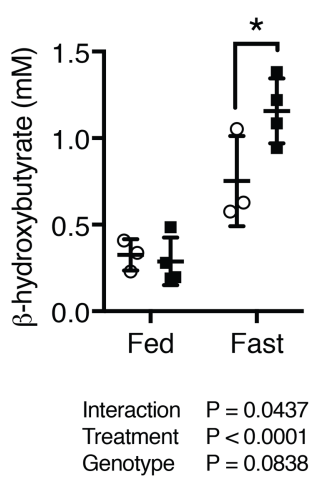

C

Cpt1a

○ AD.GFP

- AD.IQGAP1

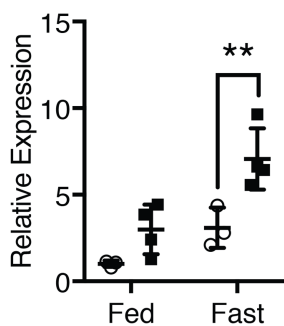

Interaction $P=0.2034$

Treatment $P=0.0018$

Genotype $\quad P=0.0022$

Figure 6. Reexpressing IQGAP1 in Iqgap1-/- livers restores appropriate mTORC1 and PPAR $\alpha$ signaling. Adenoviruses expressing either GFP or IQGAP1 were injected into female Iqgap1 $1^{-1-}$ mice via the tail vein. Two weeks later, mice were fed ad libitum or fasted 24 hours. (A) Immunoblot of fed AD.GFP and AD.IQGAP1 liver extracts. Each lane represents an individual mouse ( $n=3$ mice per group). P-S6 (S235/236) and P-S6 (S240/244) levels were normalized to total S6 expression, and p-4E-BP1 levels were normalized to GAPDH expression. The relative ratio of AD.IQCAP1 to AD.GFP mice is stated. (B) Serum $\beta$-hydroxybutyrate levels ( $n=3$ mice per group). (C and D) Hepatic gene expression of (C) Cpt1a and (D) Ehhadh, Cyp4a10, and Acadm. Expression was normalized to Gapdh expression. (E) Schematic depicting how IQGAP1 deletion affects liver physiology. Values are displayed as mean \pm SD. Two-way ANOVA with Bonferroni multiple comparisons test was used to determine significance between 2 groups under 2 conditions. ${ }^{*} P<0.05,{ }^{* *} P<0.01,{ }^{* * *} P<0.001$. See complete unedited blots in Supplemental Figure 7.

them with nutritional ketosis for 4 weeks $(41,42)$. This defect was not due to mobilization of fatty acids into the liver since hepatic triglycerides were increased in Iqgap $1^{--}$animals (Figure 2D). But the reason for lower circulating concentrations of NEFA and triglyceride levels in Iqgap $1^{-/-}$mice is therefore unclear. Overall, these data indicate downstream defects in hepatic fatty acid metabolism and ketogenesis in Iqgap $1^{1--}$ mice.

We mapped this metabolic defect in Iqgap $1^{---}$livers to impaired ligand-activated nuclear receptor PPAR $\alpha$ signaling. PPAR $\alpha$ is a primary regulator of the fasting response such that $\mathrm{Ppara}^{-1-}$ mice exhibit elevated hepatic triglycerides, reduced expression of $\beta$-oxidation genes, and lower serum FGF21 and ketone body levels during prolonged ketosis $(25,26)$. Similarly, $F g f 21^{-1-}$ mice also show lower ketone body levels when fed $\mathrm{KD}(43,44)$. Iqgap $1^{-/-}$mice exhibit an overlapping phenotype with both Ppara ${ }^{-/}$and $\mathrm{Fg} f 21^{-/-}$mice, indicating that IQGAP1 is crucial for regulating ketogenesis (Table 2). Furthermore, it has been shown that fatty acids, particularly those synthesized de novo by Fasn, act as physiological ligands for that PPAR $\alpha$ (45). Hepatic

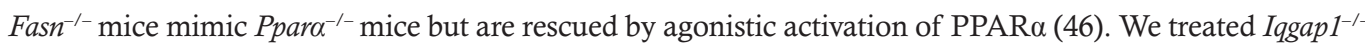
animals with the PPAR $\alpha$ synthetic agonist WY (33) and found that PPAR $\alpha$ activation did not restore all the PPAR $\alpha$ gene targets examined in this study; a subset of genes, including Ppara, Acadm, Hmgcs2, and Bdh, did not respond, whereas Cpt1a and Ehhadh were induced at levels comparable to those of WT mice. PPARa agonistic induction of Fgf21 and Cyp $4 a 10$ was higher in Iqgap $1^{--}$animals, and these data suggest that endogenous ligand may be a limiting factor for PPAR $\alpha$ activation of these genes in Iqgap $1^{-1-}$ mice.

Since we found a defective response to fasting cues in the liver, we examined the levels of the fed-state sensor mTOR in Iqgap $1^{-1-}$ mice and were surprised to find increased mTORC1 activity. Further examination during fasted and refed states revealed that deletion of IQGAP1 resulted in higher 


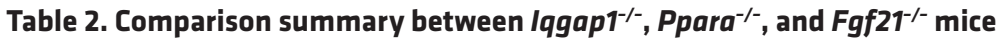

\begin{tabular}{|c|c|c|c|}
\hline Parameter & Iqgap $1^{-/-}$ & Ppara $^{-/-}$ & Fgf $21^{-1-}$ \\
\hline \multicolumn{4}{|l|}{ Fed } \\
\hline Body weight & NS & NS (54) & NS $(43,55,56)$ \\
\hline Serum NEFAs & NS & NS $(25,27) ; \uparrow(54,57)$ & NS $(25,43,55,56,58)$ \\
\hline Serum triglycerides & $\downarrow$ & NS $(25,57)$ & NS $(25,43,55,56,58)$ \\
\hline Hepatic triglycerides & NS & NS $(25,54,57)$ & NS $(25,58)$ \\
\hline Serum NEFAs & $\downarrow$ & $\uparrow(25,27,54) ;$ NS (59) & $\downarrow(43) ; \uparrow(55,56,58)$ \\
\hline Serum triglycerides & $\downarrow$ & NS $(25,59)$ & NS $(43,55,56,58)$ \\
\hline Hepatic triglycerides & NS & $\uparrow(25,54,59)$ & NS (55); $\uparrow(58)$ \\
\hline \multicolumn{4}{|l|}{$\mathrm{KD}$} \\
\hline Serum ketones & $\downarrow$ & NS (25); $\downarrow$ (57) & $\downarrow(25,43)$ \\
\hline
\end{tabular}

mTORC1 downstream kinase signaling. We also observed that transiently increasing IQGAP1 expression could suppress mTORC1 signaling in cells. This result is opposite to a previous study (47), which showed that the IR-WW domain of IQGAP1 positively regulated mTORC1 in the absence of growth factors. We attribute this difference in data to the context and experimental approach utilized. We used the livers from IQGAP1-knockout animals after nutrient deprivation, whereas the previous study used either IR-WW or C-terminal domains of IQGAP1 for their analysis. However, in line with multiple earlier reports, we did observe that IQGAP1 can directly bind to mTOR $(47,48)$.

It was shown that mTORC1 can inhibit PPAR $\alpha$ activation by recruiting the corepressor NCoR 1 to PPAR $\alpha$-response elements (21). Our data along with this finding suggest that the dampening in PPARo activation in Iqgap $1^{-/-}$mice could be secondary to the higher mTORC1 activity. To test this, we inhibited mTORC1 activity with rapamycin and validated the reduction in S6 and 4E-BP1 phosphorylation. However, rapamycin treatment was not sufficient to rescue all PPAR $\alpha$ targets and promote ketogenesis. Typically, hepatic mTORC1 acts as the major regulator of cellular energetics $(10,20)$, but we have not ruled out the role for mTORC2 in this study.

Finally, we demonstrate that reintroducing IQGAP1 in Iqgap $1^{-/-}$mice was able to rescue expression of PPAR $\alpha$ and several of its targets. Thus, we conclude that IQGAP1 functions to modulate PPAR $\alpha$ activity and subsequent control of the nutritional response to long-term ketogenesis.

\section{Methods}

Animal experiments. WT and Iqgap $1^{-/-}$mice (generated in A. Bernards's laboratory, Massachusetts General Hospital, Boston, Massachusetts, USA, and obtained from Valentina Schmidt, Stony Brook University, Stony Brook, New York, USA) maintained on a 129/SVJ background were housed in flow cages at $24^{\circ} \mathrm{C}$ on a 12/12-hour-light/dark cycle, with lights on starting at 5 AM CST, corresponding to zeitgeber time (ZT) 0 . Genotype was confirmed by PCR analysis of genomic DNA as previously described (24).

Male 16- to 20-week-old mice were used for all experiments, while 11-week-old female mice were used for adenoviral expression of IQGAP1. We used sample sizes typical for this type of work ( $n \geq 5-6$ mice per group). We randomly assigned mice of the same genotype to different treatments but were not blinded during experiments and analysis. Mice were allowed ad libitum access to food and water except during fast when they were transferred to a clean cage with access only to water. Fasting experiments were initiated at ZT4, which was approximately 9 AM ( $n=6-8$ mice per genotype). Control mice were allowed ad libitum access to food and were sacrificed at ZT4 ( $n=8-9$ mice per genotype). RF mice were given standard chow 
at ZT4 following a 24-hour fast and were sacrificed 2 hours later at ZT6 ( $n=6-9$ mice per genotype). An additional cohort of RF mice was injected with $10 \mathrm{mg} / \mathrm{kg}$ rapamycin intraperitoneally 1 hour prior to RF ( $n$ = 6-13 mice per genotype). The standard chow diet was Teklad F6 Rodent Diet (8664, Envigo), consisting of $31 \%, 19 \%$, and $50 \% \mathrm{kcal}$ from protein, fat, and carbohydrate, respectively. In contrast, the KD (TD.96355, Envigo) consisted of $9.1 \%, 90.5 \%$, and $0.4 \% \mathrm{kcal}$ from protein, fat, and carbohydrates, respectively. The KD was divided into $6-\mathrm{cm}$ dishes and frozen prior to feeding, and fresh diet was provided daily $(n=6$ mice per genotype). For fibrate treatment, WY (Cayman Chemical) was dissolved in 100\% ethanol to a final concentration of $16.5 \mathrm{mg} / \mathrm{ml}$. The WY solution or vehicle was further diluted with CO to a final concentration of $5 \mathrm{mg} / \mathrm{ml}$ and stirred under vacuum overnight to remove excess ethanol. Mice were oral gavage with either CO or WY $(50 \mathrm{mg} / \mathrm{kg}$ ) for 4 consecutive days and sacrificed on the fifth day ( $n=5-6$ mice per genotype). For hepatic expression of GFP and IQGAP1, female Iqgap ${ }^{-/-}$mice were administered high-titer adenovirus expressing either cDNA via tail vein injection. Three days after injection, mice were started on a $0.5 \mathrm{~g} / \mathrm{kg}$ doxycycline (dox) diet and sacrificed after 2 weeks ( $n=3$ mice per group). All mice were sacrificed at ZT4-6.

Blood was collected from each mouse by retro-orbital bleeding just prior to sacrifice. Sera were separated by centrifugation and stored at $-80^{\circ} \mathrm{C}$ in opaque tubes. Liver and gonadal white adipose tissues were collected and flash frozen for analysis. A portion of each tissue was also fixed in $10 \%$ formalin for histological analysis.

Octanoate challenge. Sodium octanoate $(500 \mathrm{mM})$ in sterile $\mathrm{H}_{2} \mathrm{O}$ was injected intraperitoneally at $6 \mu \mathrm{l} / \mathrm{g}$ of body weight into WT and Iqgap $1^{-1-}$ mice after a 24-hour fast. Serum ketones were measured in tail blood using a Keto-Mojo Ketone and Glucose Meter immediately before octanoate injection and 2 hours following octanoate injection ( $n=6-8$ mice per group).

Isolation and primary hepatocyte culture. Mouse hepatocytes were isolated using a 2-step collagenase perfusion technique (49). Briefly, male adult 129/SVJ WT and Iqgap1 ${ }^{-1-}$ mice were perfused with $50 \mathrm{ml}$ Solution 1 ( $1 \mu \mathrm{M}$ EDTA in $1 \times$ Hanks Balanced Salt Solution without $\mathrm{Ca}^{2+}$ or $\left.\mathrm{Mg}^{2+}\right)$. Livers were then perfused with $50 \mathrm{ml}$ Solution 2 (3,000 U collagenase type I from Worthington, $0.54 \mu \mathrm{M} \mathrm{CaCl}_{2}, 40 \mu \mathrm{g} / \mathrm{ml}$ trypsin inhibitor, and 15 mM HEPES, $\mathrm{pH} 7.4$, in $1 \times$ Hanks Balanced Salt Solution with $\mathrm{Ca}^{2+}$ and $\mathrm{Mg}^{2+}$ ). The perfused liver was then transferred to a Petri dish containing wash buffer (Williams E media with $1 \times$ penicillin/streptomycin and $1 \times$ L-glutamine) and gently massaged to loosen liver cells. The cell suspension was filtered through a 70- $\mu \mathrm{m}$ filter. For isolation of hepatocytes and nonparenchymal cells (NPCs), the cell suspension was centrifuged twice at $50 \mathrm{~g}$ for 10 minutes. The supernatant was transferred to a new tube and centrifuged at $680 \mathrm{~g}$ to isolate NPCs. The pellet contained hepatocytes. The isolated hepatocytes and NPCs were incubated in red blood cell lysis buffer followed by 3 washes with $1 \times$ PBS with 10-minute spins at $320 \mathrm{~g}$ and $680 \mathrm{~g}$, respectively, after each wash. For primary cell culture, the cell suspension was centrifuged at $600 \mathrm{~g}$ for 4 minutes. The pellet was suspended in $25 \mathrm{ml}$ wash buffer and layered onto a Percoll gradient and immediately centrifuged at $600 \mathrm{~g}$ for 10 minutes. The pellet, enriched for live hepatocytes, was washed 3 times and then cells were suspended in growing medium (wash media supplemented with $1 \times$ Insulin-Transferrin-Selenium [ITS] solution from Gibco) and plated at $5 \times 10^{5}$ live cells $/ \mathrm{ml}$ in 6 -well collagen-coated plates. Media were changed 4-6 hours after plating to remove dead cells. Cells were either cultured for another 24 hours in growing medium.

Adenovirus production and cell culture. HepG2 cell line was obtained from ATCC (catalog HB-8065) and cultured according to ATCC specifications. This cell line tested negative for mycoplasma (Biotool, cata$\log$ B39032). For in vivo infection, adenoviruses expressing full-length Iqgap1 and Gfp were generated as previously described (50). For IQGAP1 overexpression in cell culture, HepG2 cells were cultured in tetracycline-free DMEM and transfected with tetracycline-inducible FLAG-tagged Iqgap1 and rtTA plasmids using a Mirus TransIT-X2 kit. The next day, the media were replaced with fresh DMEM containing 2 ng/ $\mathrm{ml}$ dox, and protein was collected 24 hours later. For glucose and serum starvation experiments, HepG2 cells were cultured with HBSS supplemented with 10\% FBS, $2 \mathrm{mM}$ glutamine, and $10 \mathrm{U} / \mathrm{ml}$ penicillin and streptomycin for 12 hours. After this, the HepG2 cells were switched to HBSS media lacking either glucose or FBS or both for 6 hours before analysis.

Serum chemistry. Sera were thawed on ice prior to each metabolite assay. Assays were run in duplicate for triglycerides (Infinity Triglyceride Stable Reagent, Fisher Scientific), $\beta$-hydroxybutyrate ( $\beta$-Hydroxybutyrate (Ketone Body) Colorimetric Assay Kit, Cayman Chemical), and free fatty acids (Free Fatty Acid Fluorometric Assay Kit, Cayman Chemical). All assays were performed according to the kit instructions, unless stated otherwise. For the Free Fatty Acid Kit, absorbance was read at $570 \mathrm{~nm}$ and 
was adjusted for background reading at $600 \mathrm{~nm}$. Additionally, Mouse/Rat FGF-21 Quantikine ELISA kit (R\&D Systems) was used to measure serum FGF21 levels. Serum glucose was measured using onetouch glucose strips on fresh tail blood.

Glucose tolerance test. Glucose tolerance test was performed as previously described (51). Briefly, mice were fasted for 13 hours overnight and administered $2 \mathrm{~g} / \mathrm{kg}$ D-glucose intraperitoneally. Blood glucose concentrations were measured at $0,15,30,60$, and 120 minutes after injection using tail blood.

Hepatic triglyceride assay. Frozen liver tissue ( $<50 \mathrm{mg}$ ) was weighed and homogenized in $1 \mathrm{ml}$ isopropanol. Samples were centrifuged at $4^{\circ} \mathrm{C}$ and $9,931 \mathrm{~g}$ for 15 minutes, and supernatant containing triglycerides was separated. Triglyceride content was measured using Infinity Triglyceride Liquid Stable Reagent (Fisher Scientific).

Histology. Formalin-fixed liver samples were embedded in paraffin wax. Five-micron sections were cut and used for H\&E staining according to standard methods (52). For Oil red O staining, additional liver samples were frozen in OCT media upon collection and cut into 8-micron sections. Once dried, the sections were fixed in $10 \%$ formalin, rinsed with running tap water then $60 \%$ isopropanol, and stained with fresh Oil red $\mathrm{O}$ working solution ( $0.15 \mathrm{~g}$ Oil red $\mathrm{O}$ dye in $50 \mathrm{ml}$ of $60 \%$ isopropanol) for 15 minutes. The slides were then rinsed with 60\% isopropanol, stained using Modified Harris Hematoxylin (Richard Allan, 72711) for 3 minutes, rinsed with $\operatorname{diH}_{2} \mathrm{O}$, and cover slipped.

Quantitative RT-PCR. Total RNA from frozen whole liver tissue was extracted using TRIzol solution (Invitrogen) according to the manufacturer's protocol. RNA quality was determined by A260/280 and bleach RNA gel as previously described (53). RNA (5 $\mu$ g) was treated with DNase (Promega) and reverse transcribed using random hexamer primers (New England Biosciences) and the Maxima Reverse Transcriptase kit (Thermo Fisher Scientific). The cDNA was diluted to $12.5 \mathrm{ng} / \mu 1$ with molecular-grade water (Corning) and used for qRT-PCR assays. qRT-PCR was performed on an Eco Real-Time PCR system (Illumina) in triplicate using $50 \mathrm{ng}$ cDNA per reaction and PerfeCTa SYBR Green FastMix (Quanta). All assays were run with an initial activation step for 10 minutes at $95^{\circ} \mathrm{C}$, followed by 40 cycles of $95^{\circ} \mathrm{C}$ for 10 seconds and $60^{\circ} \mathrm{C}$ for 30 seconds. Primer sequences are described in Supplemental Table 1. Gapdh, $\beta$-actin, and 3664 were used as housekeeping genes.

Western blot analysis. Protein was extracted from approximately $50 \mathrm{mg}$ frozen liver tissue. RIPA buffer (25 mM Tris, pH 7, $150 \mathrm{mM} \mathrm{NaCl}, 0.5 \%$ sodium deoxycholate, protease inhibitor [Pierce Protease Inhibitor Mini Tablets, EDTA Free; Thermo Fisher Scientific], phosphatase inhibitor [Phosphatase Inhibitor Cocktail 3; MilliporeSigma], and 1\% Triton X-100) was added to each liver sample, which was homogenized by adding eight to ten $1-\mathrm{mm}$ beads and bullet blending for 3 times for 1 minute each time, with 1 minute on ice in between. Samples were sonicated until nonviscous. Protein concentration was measured by BCA assay (Pierce BCA Protein Assay Kit, Thermo Fisher Scientific). For Western blot, $10-50 \mu \mathrm{g}$ of total protein was loaded onto $8 \%-12 \%$ SDS-PAGE gels. After transfer, the membrane was incubated with antibodies described in Supplemental Table 2. See complete unedited blots in Supplemental Figure 7.

Coimmunoprecipitation. HepG2 cells were transfected with dox-inducible FLAG-IQGAP1 and were treated with dox or vehicle for 24 hours. After induction, approximately $10^{7}$ cells were lysed in lysis buffer (50 mM Tris, pH 7.8, $150 \mathrm{mM} \mathrm{NaCl}, 30 \mathrm{mM}$ EDTA, 0.5\% Triton X-100). The lysate was then split and equal amounts were immunoprecipitated with a FLAG antibody overnight, washed, and resolved on an $8 \%$ SDS-PAGE gel. After transfer, blots were probed with antibodies for mTOR.

Statistics. Data are expressed as mean \pm SD. All statistical analyses were performed using GraphPad Prism software version 6. Student's unpaired 2-tailed $t$ test was used to compare 2 groups. Two-way ANOVA with Bonferroni multiple comparisons test was performed to compare two groups with two treatments. Asterisks indicate a statistically significant difference between the treatment group and its respective genotype control unless the groups are otherwise indicated. Significance is defined as $P<0.05$. Outliers were determined by Grubbs' test and removed from analysis.

Study approval. All animal studies were approved by the University of Illinois at Urbana-Champaign Institutional Animal Care and Use Committee and were carried out as outlined in the Guide for the Care and Use of Laboratory Animals (National Academies Press, 2011).

\section{Author contributions}

HLE and SA contributed to the conception, design, data acquisition, analysis, and drafting of the manuscript. 


\section{Acknowledgments}

The authors would like to thank Andre Bernards and Valentina Schmidt for sharing the Iqgap ${ }^{-/-}$mice. We would also like to thank Jie Chen, Professor of Cell and Developmental Biology at the University of Illinois at Urbana-Champaign and Shomit Sengupta, Senior Scientist, Navitor Pharmaceuticals, Massachusetts, USA, for their critical comments and suggestions during this study. We thank Karen Wendt who initiated the fasting experiments in Iqgap $1^{-/-}$mice. This study was supported by start-up funds from University of Illinois at Urbana-Champaign (to SA), National Institute of Child Health and Human Development grant HD080011 (to SA), and National Cancer Institute grant F30CA206495 (to HLE).

Address correspondence to: Sayeepriyadarshini Anakk, University of Illinois at Urbana-Champaign, 4453 Medical Sciences Building, 506 S Mathews Ave., Urbana, Illinois, USA. Phone: 217.300.7905; Email: anakk@illinois.edu.

1. Briggs MW, Sacks DB. IQGAP1 as signal integrator: $\mathrm{Ca}^{2+}$, calmodulin, Cdc42 and the cytoskeleton. FEBS Lett. 2003;542(1-3):7-11.

2. Smith JM, Hedman AC, Sacks DB. IQGAPs choreograph cellular signaling from the membrane to the nucleus. Trends Cell Biol. 2015;25(3):171-184

3. Kuroda S, et al. Role of IQGAP1, a target of the small GTPases Cdc42 and Rac1, in regulation of E-cadherin- mediated cellcell adhesion. Science. 1998;281(5378):832-835

4. Mataraza JM, Briggs MW, Li Z, Entwistle A, Ridley AJ, Sacks DB. IQGAP1 promotes cell motility and invasion. J Biol Chem. 2003;278(42):41237-41245.

5. Roy M, Li Z, Sacks DB. IQGAP1 binds ERK2 and modulates its activity. J Biol Chem. 2004;279(17):17329-17337.

6. Roy M, Li Z, Sacks DB. IQGAP1 is a scaffold for mitogen-activated protein kinase signaling. Mol Cell Biol. 2005;25(18):7940-7952.

7. Chawla B, Hedman AC, Sayedyahossein S, Erdemir HH, Li Z, Sacks DB. Absence of IQGAP1 protein leads to insulin resistance. J Biol Chem. 2017;292(8):3273-3289.

8. Choi S, Hedman AC, Sayedyahossein S, Thapa N, Sacks DB, Anderson RA. Agonist-stimulated phosphatidylinositol-3,4,5-trisphosphate generation by scaffolded phosphoinositide kinases. Nat Cell Biol. 2016;18(12):1324-1335.

9. Cahill GF Jr. Fuel metabolism in starvation. Annu Rev Nutri. 2006;26:1-22.

10. Cornu M, Albert V, Hall MN. mTOR in aging, metabolism, and cancer. Curr Opin Genet Dev. 2013;23(1):53-62.

11. Evans RM, Barish GD, Wang YX. PPARs and the complex journey to obesity. Nat Med. 2004;10(4):355-361.

12. Kersten S, Desvergne B, Wahli W. Roles of PPARs in health and disease. Nature. 2000;405(6785):421-424.

13. Laplante M, Sabatini DM. mTOR signaling in growth control and disease. Cell. 2012;149(2):274-293.

14. Yecies JL, et al. Akt stimulates hepatic SREBP1c and lipogenesis through parallel mTORC1-dependent and independent pathways. Cell Metab. 2011;14(1):21-32.

15. Cherrington AD. Banting Lecture 1997. Control of glucose uptake and release by the liver in vivo. Diabetes. 1999;48(5):1198-1214.

16. Krebs HA. The regulation of the release of ketone bodies by the liver. Adv Enzyme Regul. 1966;4:339-354.

17. Kersten S. Integrated physiology and systems biology of PPAR $\alpha$. Mol Metab. 2014;3(4):354-371.

18. Reddy JK, Hashimoto T. Peroxisomal $\beta$-oxidation and peroxisome proliferator-activated receptor $\alpha$ : an adaptive metabolic system. Annu Rev Nutr. 2001;21:193-230.

19. Howell JJ, Manning BD. mTOR couples cellular nutrient sensing to organismal metabolic homeostasis. Trends Endocrinol Metab. 2011;22(3):94-102.

20. Saxton RA, Sabatini DM. mTOR signaling in growth, metabolism, and disease. Cell. 2017;169(2):361-371.

21. Sengupta S, Peterson TR, Laplante M, Oh S, Sabatini DM. mTORC1 controls fasting-induced ketogenesis and its modulation by ageing. Nature. 2010;468(7327):1100-1104.

22. Gwinn DM, et al. AMPK phosphorylation of raptor mediates a metabolic checkpoint. Mol Cell. 2008;30(2):214-226.

23. Jameson KL, et al. IQGAP1 scaffold-kinase interaction blockade selectively targets RAS-MAP kinase-driven tumors. Nat Med. 2013;19(5):626-630.

24. Li S, Wang Q, Chakladar A, Bronson RT, Bernards A. Gastric hyperplasia in mice lacking the putative Cdc42 effector IQGAP1 Mol Cell Biol. 2000;20(2):697-701.

25. Badman MK, Pissios P, Kennedy AR, Koukos G, Flier JS, Maratos-Flier E. Hepatic fibroblast growth factor 21 is regulated by PPARalpha and is a key mediator of hepatic lipid metabolism in ketotic states. Cell Metab. 2007;5(6):426-437.

26. Inagaki T, et al. Endocrine regulation of the fasting response by PPAR $\alpha$-mediated induction of fibroblast growth factor 21 . Cell Metab. 2007;5(6):415-425.

27. Kersten S, Seydoux J, Peters JM, Gonzalez FJ, Desvergne B, Wahli W. Peroxisome proliferator-activated receptor $\alpha$ mediates the adaptive response to fasting. J Clin Invest. 1999;103(11):1489-1498.

28. Muerhoff AS, Griffin KJ, Johnson EF. The peroxisome proliferator-activated receptor mediates the induction of CYP4A6, a cytochrome P450 fatty acid omega-hydroxylase, by clofibric acid. J Biol Chem. 1992;267(27):19051-19053.

29. Patsouris D, Reddy JK, Müller M, Kersten S. Peroxisome proliferator-activated receptor $\alpha$ mediates the effects of high-fat diet on hepatic gene expression. Endocrinology. 2006;147(3):1508-1516.

30. Rodríguez JC, Gil-Gómez G, Hegardt FG, Haro D. Peroxisome proliferator-activated receptor mediates induction of the mitochondrial 3-hydroxy-3-methylglutaryl-CoA synthase gene by fatty acids. J Biol Chem. 1994;269(29):18767-18772.

31. Schoonjans K, et al. PPAR $\alpha$ and PPAR $\gamma$ activators direct a distinct tissue-specific transcriptional response via a PPRE in the lipoprotein lipase gene. EMBO J. 1996;15(19):5336-5348.

32. Hashimoto T, Cook WS, Qi C, Yeldandi AV, Reddy JK, Rao MS. Defect in peroxisome proliferator-activated receptor $\alpha$-inducible 
fatty acid oxidation determines the severity of hepatic steatosis in response to fasting. J Biol Chem. 2000;275(37):28918-28928.

33. Green S. PPAR: a mediator of peroxisome proliferator action. Mutat Res. 1995;333(1-2):101-109.

34. Brunn GJ, et al. Phosphorylation of the translational repressor PHAS-I by the mammalian target of rapamycin. Science. 1997;277(5322):99-101.

35. Burnett PE, Barrow RK, Cohen NA, Snyder SH, Sabatini DM. RAFT1 phosphorylation of the translational regulators p70 S6 kinase and 4E-BP1. Proc Natl Acad Sci US A. 1998;95(4):1432-1437.

36. Porstmann T, et al. SREBP activity is regulated by mTORC1 and contributes to Akt-dependent cell growth. Cell Metab. 2008;8(3):224-236.

37. McGarry JD, Mannaerts GP, Foster DW. A possible role for malonyl-CoA in the regulation of hepatic fatty acid oxidation and ketogenesis. J Clin Invest. 1977;60(1):265-270.

38. Hart MJ, Callow MG, Souza B, Polakis P. IQGAP1, a calmodulin-binding protein with a rasGAP-related domain, is a potential effector for cdc42Hs. EMBO J. 1996;15(12):2997-3005.

39. Pan CW, et al. AKT-phosphorylated FOXO1 suppresses ERK activation and chemoresistance by disrupting IQGAP1-MAPK interaction. EMBO J. 2017;36(8):995-1010.

40. Yoon JC, et al. Control of hepatic gluconeogenesis through the transcriptional coactivator PGC-1. Nature. 2001;413(6852):131-138.

41. Bielohuby $\mathrm{M}$, et al. Induction of ketosis in rats fed low-carbohydrate, high-fat diets depends on the relative abundance of dietary fat and protein. Am J Physiol Endocrinol Metab. 2011;300(1):E65-E76.

42. Kennedy AR, et al. A high-fat, ketogenic diet induces a unique metabolic state in mice. Am J Physiol Endocrinol Metab. 2007;292(6):E1724-E1739.

43. Badman MK, Koester A, Flier JS, Kharitonenkov A, Maratos-Flier E. Fibroblast growth factor 21-deficient mice demonstrate impaired adaptation to ketosis. Endocrinology. 2009;150(11):4931-4940.

44. Puchalska P, Crawford PA. Multi-dimensional roles of ketone bodies in fuel metabolism, signaling, and therapeutics. Cell Metab. 2017;25(2):262-284.

45. Chakravarthy MV, et al. Identification of a physiologically relevant endogenous ligand for PPAR $\alpha$ in liver. Cell. 2009;138(3):476-488.

46. Chakravarthy MV, et al. "New" hepatic fat activates PPARalpha to maintain glucose, lipid, and cholesterol homeostasis. Cell Metab. 2005;1(5):309-322.

47. Tekletsadik YK, Sonn R, Osman MA. A conserved role of IQGAP1 in regulating TOR complex 1. J Cell Sci. 2012;125(pt 8):2041-2052

48. Chen F, Zhu HH, Zhou LF, Wu SS, Wang J, Chen Z. IQGAP1 is overexpressed in hepatocellular carcinoma and promotes cell proliferation by Akt activation. Exp Mol Med. 2010;42(7):477-483.

49. Li WC, Ralphs KL, Tosh D. Isolation and culture of adult mouse hepatocytes. Methods Mol Biol. 2010;633:185-196

50. Anakk S, Bhosale M, Schmidt VA, Johnson RL, Finegold MJ, Moore DD. Bile acids activate YAP to promote liver carcinogenesis. Cell Rep. 2013;5(4):1060-1069.

51. Norris AW, et al. Muscle-specific PPAR $\gamma$-deficient mice develop increased adiposity and insulin resistance but respond to thiazolidinediones. J Clin Invest. 2003;112(4):608-618

52. Cardiff RD, Miller CH, Munn RJ. Manual hematoxylin and eosin staining of mouse tissue sections. Cold Spring Harb Protoc. 2014;2014(6):655-658.

53. Aranda PS, LaJoie DM, Jorcyk CL. Bleach gel: a simple agarose gel for analyzing RNA quality. Electrophoresis. 2012;33(2):366-369.

54. Montagner A, et al. Liver PPARalpha is crucial for whole-body fatty acid homeostasis and is protective against NAFLD. Gut. 2016;65(7):1202-1214

55. Hotta Y, et al. Fibroblast growth factor 21 regulates lipolysis in white adipose tissue but is not required for ketogenesis and triglyceride clearance in liver. Endocrinology. 2009;150(10):4625-4633.

56. Potthoff MJ, et al. FGF21 induces PGC-1alpha and regulates carbohydrate and fatty acid metabolism during the adaptive starvation response. Proc Natl Acad Sci U S A. 2009;106(26):10853-10858.

57. Oishi K, Uchida D, Ohkura N, Horie S. PPARalpha deficiency augments a ketogenic diet-induced circadian PAI-1 expression possibly through PPARgamma activation in the liver. Biochem Biophys Res Commun. 2010;401(2):313-318.

58. Antonellis PJ, Hayes MP, Adams AC. Fibroblast growth factor 21-null mice do not exhibit an impaired response to fasting. Front Endocrinol (Lausanne). 2016;7:77.

59. Nakagawa Y, et al. CREB3L3 controls fatty acid oxidation and ketogenesis in synergy with PPARalpha. Sci rep. 2016;6:39182. 